\title{
Publisher Correction: Classic rational bubbles and representativeness
}

\author{
Massimiliano Ferrara $^{1}$ (D) Bruno Antonio Pansera ${ }^{1}$. \\ Francesco Strati ${ }^{1}$
}

\section{Correction to: Decisions Econ Finan (2018) 41:19-34 https://doi.org/10.1007/s10203-018-0205-4}

In the original publication, the copyright holder was incorrectly published as 'SpringerVerlag Italia S.r.l., part of Springer Nature' instead of 'Associazione per la Matematica Applicata alle Scienze Economiche e Sociali (AMASES)'.

The original article can be found online at https://doi.org/10.1007/s10203-018-0205-4.

$\bowtie \quad$ Massimiliano Ferrara massimiliano.ferrara@unirc.it

Bruno Antonio Pansera bruno.pansera@unirc.it

Francesco Strati francesco.strati@unirc.it

1 Department of Law and Economics, University Mediterranea of Reggio Calabria, Via dei Bianchi, 2, 89127 Reggio Calabria, Italy 